\title{
Acute Inflammatory Response to a Single Bout of Resistance Exercise with or Without Blood Flow Restriction
}

\author{
Mohammad Bani Asadi ${ }^{1}$, Hassan Sharifi ${ }^{1}$, Bahram Abedi (iD ${ }^{1,{ }^{*}}$ and Hoseyn Fatolahi (iD) ${ }^{2}$ \\ ${ }^{1}$ Department of Physical Education and Sport Sciences, Mahallat Branch, Islamic Azad University, Mahallat, Iran \\ ${ }^{2}$ Department of Physical Education, Pardis Branch, Islamic Azad University, Pardis, Iran \\ "Corresponding author: Department of Physical Education and Sport Sciences, Mahallat Branch, Islamic Azad University, Mahallat, Iran. Email: abedi@iaumahallat.ac.ir \\ Received 2020 October 25; Accepted 2021 February 27.
}

\begin{abstract}
Background: The effectiveness of resistance training (RT) with blood flow restriction (BFR) is limited from the health perspective. Objectives: This study aimed to investigate the acute inflammatory response to a single bout of RT with or without BFR.

Methods: Twenty-four non-athletic male students were randomly divided into 3 groups ( $\mathrm{n}=8$ ), including: (1) high intensity (HIRT$80 \%$ of 1 -RM: $3 \times 10 \mathrm{R}),(2)$ low intensity $(40 \%$ of $1-\mathrm{RM}: 3 \times 30 \mathrm{R})$ with BFR $(180 \mathrm{mmHg})(\mathrm{LIRT}+\mathrm{BFR})$ and $(3)$ LIRT.

Results: Significant increase for IL1 $\beta$ ( $\mathrm{p}=0.001)$, IL-6 ( $\mathrm{P}=0.001)$, TNF- $\alpha(\mathrm{P}=0.002), \mathrm{CRP}(\mathrm{P}=0.007), \operatorname{ICAM}(\mathrm{P}=0.016), \mathrm{HCY}(\mathrm{P}=0.008)$ were observed. These responses in the HIRE and LIRT + BFR groups were significantly higher than in the LIRT group respectively (P $\leq 0.05)$.

Conclusions: The LIRT + BFR has similar effects to HIRT and can be used in rehabilitation training. The gradual overload and proper recovery are very important due to the increase in acute inflammatory responses.
\end{abstract}

Keywords: Sports Medicine, Exercise Science, Rehabilitation, Exercise Mode, Athletic Therapy

\section{Background}

In many groups, especially adolescent boys, resistance training is considered an exercise mode. Resistance training increases muscle (hypertrophy) and reduces inflammatory indicators $(1,2)$. However, in response to a session of resistance exercise, a circular increase in inflammatory biomarkers has been observed (3). Blood flow restriction (BFR) is an exercise mode of resistance training. Even light activities using blood flow restriction (Katsu's light resistance training) have increased muscle strength, hypertrophy and improved physiological indicators or prevented atrophy in healthy and diseased groups (4-6). The use of low-intensity training with BFR is also appropriate for rehabilitation, as well as in the elderly who are unable to exercise vigorously (4-11). Training with BFR is such that the proximal part of the training limb is tied with a band of tape or cuff(tourniquet), thus restricting blood flow to the target area $(5,6)$.

Resistance training, especially in the form of BFR, has been shown to reduce oxygen delivery and increase oedema in the position used and this can increase acute inflammation $(12,13)$. Also, a decrease in tissue blood flow is one of the factors that increase inflammatory markers $(14,15)$. However, adapting to regular exercise in healthy and patient groups improves inflammatory rest levels and reduces inflammatory responses such as IL1- $\beta$, IL-6, TNF- $\alpha$, CRP, sICAM1 and homocysteine (HCY) the increase in acute inflammation caused by a session of exercise activity at different intensities, especially in untrained groups or groups with risk factors, has been confirmed (12,16-19).

One of the most important functions of the immune system is to produce soluble or cellular components called cytokines, which protect the body against any inflammatory agents. The release of cytokines, such as IL-1 and IL6 , as general and effective regulatory factors in inflammatory responses, stimulates the production and secretion of C-reactive protein (CRP) and fibrinogen from the liver (19). CRP measurement is the best way to diagnose tissue inflammation due to its rapid increase at the onset of tissue inflammation and its rapid decrease as soon as it heals (20). CRP is a plasma soluble glycoprotein that acts after infection and inflammation. Homocysteine is an amino acid that is converted to cysteine in the body $(19,20)$. If homocysteine cannot be converted to cysteine or methionine, the level of homocysteine will increase. Elevated homocys- 
teine can damage the endothelium and lead to cardiovascular disease at an early age. An increase in CRP is associated with an increase in SICAM-1 and homocysteine, indicating acute inflammation (21).

According to the principles of prescribing exercise, everyone should be familiar with the effective aspects of exercise before starting physical activity and prevent its risk factors. However, this issue is often examined only in sick people, and failure to do so may lead to some risks for young people. Regular exercise will usually create physiological adaptations. But it seems necessary to study the acute response to new methods of exercise to prevent injury. Also, the importance of acute inflammatory response is revealed when we note that some young people who engage in resistance activity may have some risk factors or congenital disorders that may be compromised in response to increased inflammatory markers due to training intensity or exercise mode such as BFR. Inflammatory acute responses to resistance exercise in the form of BFR have also been less studied, and it is very difficult to achieve an integrated finding due to differences in performing methods or groups participating in studies.

\section{Objectives}

The present study aimed to investigate the acute inflammatory response to a single bout of resistance exercise with or without blood flow restriction.

\section{Methods}

In the present study, a semi-experimental method was used to implement the research design. The participants in the study were male students who had no training, no smoking, no high blood pressure or other cardiovascular diseases, and no supplements for at least six months. Examinations were performed by a specialist physician in the Central Clinic of Markazi province, Mahallat.

Twenty four students who volunteered for the study were randomly divided into three groups including (1) lowintensity resistance training (LIRT), (2) low-intensity resistance training with blood flow restriction (LIRT + BFR) (180 $\mathrm{mmHg}$ ) and (3) high-intensity resistance training (HIRT). The details of the participants of each group are presented in Table 1.

\subsection{Resistance Exercise and BFR Protocol}

First, one maximum repetition (1- RM) was measured and recorded using the formula:
$1-R M=\frac{\text { selective weight value }}{1.0278-(\text { number of repetitions } \times 1.0278)}$

To induce blood flow restriction, a tourniquet equipped with a blood pressure monitor (54 mm wide; KAATSU Master Mini, Sato Sports Plaza, Tokyo, Japan) was used. Systolic blood pressure was adjusted to limit blood flow in the $180 \mathrm{~mm} \mathrm{Hg}$ range (5, 6).

Low-intensity resistance exercise group without blood flow restriction: This group with $40 \%$ of $1 \mathrm{RM}$ performed four squats, chest presses, forearms and forelegs for 30 repetitions and three sets with a rest interval of 1 to 2 minutes. All exercises were done in the gym of the Islamic Azad University, Mahallat Branch, from 4 to 5:30 p.m. Lowintensity resistance exercise group with blood flow restriction: This group with $40 \%$ of $1 \mathrm{RM}$ performed four squats, chest presses, forearms and forelegs for 30 repetitions and three sets with a rest interval of 1 to 2 minutes.

High-intensity resistance exercise group without blood flow restriction: This group with $80 \%$ of 1 RM performed four squats, chest presses, forearms and forelegs for 10 repetitions and three sets with a rest interval of 1 to 2 minutes.

\subsection{Laboratory Methods}

Body mass index was calculated in $\mathrm{kg} / \mathrm{m}^{2}$ using BMI equation through measuring height (Seca 213, Germany recorded to the nearest $0.1 \mathrm{~cm}$.) and weight (SECA Digital Scale Model 727: with a precision of $2 \mathrm{~g}$ ). To measure the study variables at plasma levels, fasting blood samples were collected in a sedentary position from the brachial vein before and immediately after a session of resistance exercise in each group of participants. In the first phase, all subjects were asked not to engage in any strenuous physical activity for two days before the test. The plasma concentration of IL1- $\beta$ (Biovendor Laboratorial kit, Biovendor Company, Austria), IL-6 and TNF- $\alpha$ (Bender MedSystems), CRP (hs-CRP kit, IBL, Germany), sICAM1 (DRG Instruments GmbH, Marburg, Germany), homocysteine (ELISAElabscience Biotechnology Co., Ltd., Wuhan, P. R. C. with Lot) were measured manufacturer's manual and based on the ELISA method. All experiments were performed city by the laboratory technician in the laboratory of the Central Clinic of Markazi province, Mahallat.

\subsection{Statistical Analysis}

To test the normality of data distribution, the SpiroWilk test was used. The data were calculated as the mean and standard deviation. For inferential analysis of data and to eliminate the effect of pre-test, analysis of covariance 


\begin{tabular}{lccc}
\hline \multicolumn{2}{l}{ Table 1. Characteristics of the Participants and Values of Physiological Indices Measured in the Study Groups } & \\
\hline Groups & Low-intensity RT & Low-intensity BFR RT & High-intensity RT \\
\hline Age & $24.8 \pm 3.09$ & $24.37 \pm 2.55$ & $25.7 \pm 2.91$ \\
Height $(\mathbf{c m})$ & $175.5 \pm 4.17$ & $173.6 \pm 4.2$ & $171.2 \pm 2.65$ \\
Weight $(\mathbf{K g})$ & $75 \pm 6.32$ & $73.34 \pm 8.7$ & $74.6 \pm 6.67$ \\
BMI $\left(\mathbf{K g} / \mathbf{m}^{2}\right)$ & $24.43 \pm 2.54$ & $24.45 \pm 3.14$ & $25.42 \pm 2.4$ \\
\hline
\end{tabular}

(ANCOVA) and Tukey's post hoc tests at significance level $\mathrm{P}$ $\leq 0.05$ were used applying SPSS 21 software.

\section{Results}

The comparison between the pre-test and post-test values showed an increase in IL1- $\beta$, IL-6, TNF- $\alpha$, CRP, sICAM1, HCY values compared to the pre-test levels (Figure 1).The results showed significant differencesamongthe studied groups for variables inclouding IL1- $\beta(\mathrm{P}=0.001, \mathrm{~F}=16.52$, $\eta=0.418)$, IL-6 $(\mathrm{P}=0.001, \mathrm{~F}=16.714, \eta=0.504), \mathrm{TNF}-\alpha(\mathrm{P}$ $=0.002, \mathrm{~F}=8.229, \eta=0.387), \mathrm{CRP}(\mathrm{P}=0.007, \mathrm{~F}=6.371, \eta$ $=0.349)$, ICAM1 $(\mathrm{P}=0.016, \mathrm{~F}=5.035, \eta=0.321), \mathrm{HCY}(\mathrm{P}=$ $0.008, \mathrm{~F}=6.192, \eta=0.337)$. The results of Tukey's post hoc test showed that the acute inflammatory responses in the HIRT and LIRT + BFR groups were significantly higher than in the LIRT group $(\mathrm{P} \leq 0.05)$. However, there was no significant difference between the HIRT group and the LIRT + BFR group (Figure 1).

\section{Discussion}

The present study aimed to investigate the acute inflammatory response to a single bout of resistance exercise with or without blood flow restriction. The acute inflammatory responses in the HIRT and LIRT + BFR groups were significantly higher than in the LIRT group. These changes were further influenced by the intensity of training. On the other hand, at equal intensities, the exercise mode increased the inflammatory response as BFR.

In general, adapting to exercise training reduces the baseline levels of inflammatory markers, but increase in response to a session of exercise $(16,18,19,22)$. Previous studies have shown that LIRT+BFR has the best functional and health effects $(2,8,10)$. There was also no significant difference in the performance indicators between highintensity and medium-intensity BFR cycling training (7). The findings of the present study show that the use of LIRT+BFR has less inflammatory consequences and is recommended to achieve good health. LIRT + BFR can increase muscle strength and volume without stimulating inflammatory pathways such as CRP (20).

Changes in inflammatory cytokines levels during exercise depend on age, gender, fitness, duration, intensity, type of activity, type of muscle fibre, and muscle mass used in mechanical work. Besides training status, metabolic stress, training load, rest time, repetitions, and training volume have been identified as effective training components for muscle adaptation and acute hormonal responses during resistance training (22).

The high-pressure BFR exercises reduce oxygen delivery and increase metabolic stress (12). One of the most important mechanisms for the effectiveness of BFR exercise is the creation of hypoxia and ischemia (23). Therefore increase in inflammation caused by BFR exercise can be due to tissue reperfusion involved (24). It was noted that decreased tissue blood flow and subsequent hypoxia alone could also increase inflammation $(14,15)$. Due to the decrease in blood flow, BFR training slows the lactate produced inside the cell causes a stronger acidic environment (One of the stimulants of testosterone secretion).

One of the effective ways of BFR training is to strengthen the antioxidant pathways and improve the performance of the sodium-potassium pump associated with ATP (7). Stimulation of anabolic pathways such as mTOR, NO, heat shock proteins (HSPs), and myostatin is also among other signaling pathways under the influence of blood flow restriction due to hypoxia and ischemia (25). The intensity of exercise through its influence on different hemodynamic, metabolic, immunological, and endocrine pathways is still one of the most important reasons for the increase in acute inflammatory responses to exercise (21, 26).

It has been stated that inflammatory responses such as CRP or sICAM have no significant relationship with physiological and anthropometric indicators and have shown independent changes $(27,28)$. On the other hand, an increase in some inflammatory indicators, especially IL-6, is associated with a decrease in energy reserves, which can also affect eating habits after exercise (16). In confirmation of the effect of metabolic stress on increasing inflamma- 
A

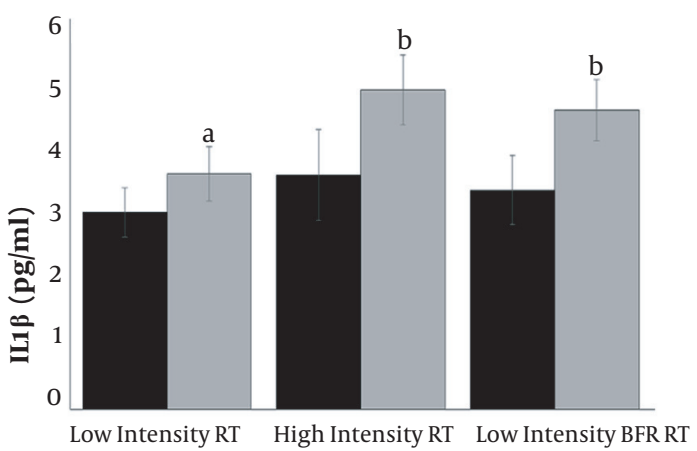

B

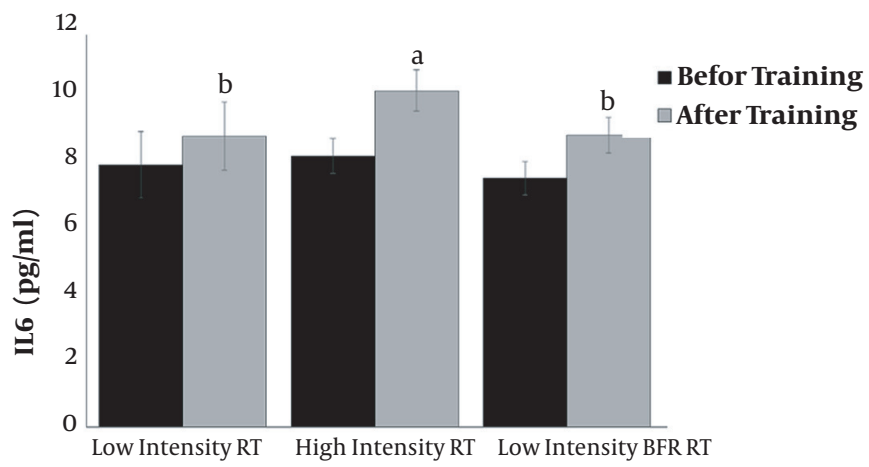

C

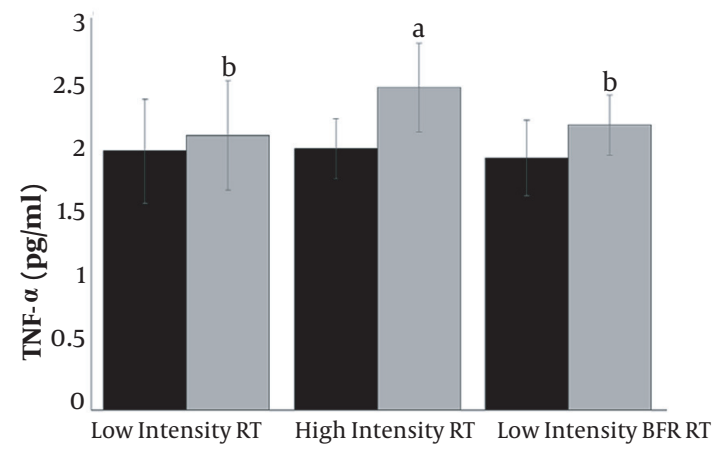

D

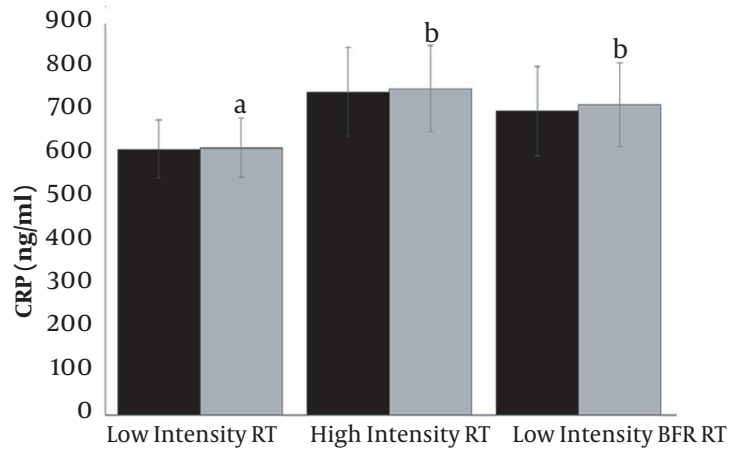

E

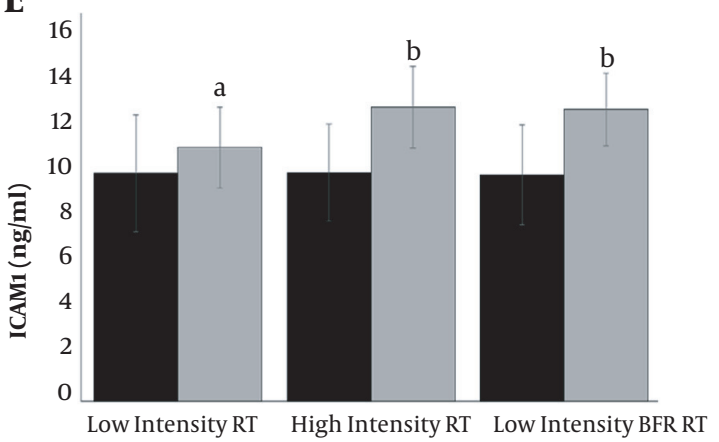

F

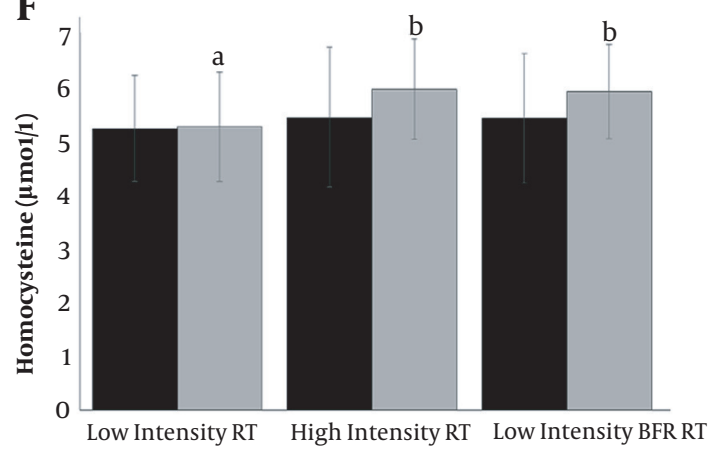

Figure 1. Changes in the inflammatory biomarkers in response to a single bout of resistance exercise with or without blood flow restriction. The data presented as mean and standard divination. Similar alphabetical letters indicate the same changes and different alphabetic letters indicate significant changes. (RT: Resistance training, BFR: Blood flow restriction)

tory biomarker, it has been shown that increased homocysteine may be due to increased creatine breakdown to provide ATP, although increased IL-6 may also be affected by increased CRP (3). Following these changes, an increase in TNF- $\alpha$ can also interfere with the entry of glucose into the muscle cell, which is one of the causes of fatigue and increased metabolic stress, followed by increased inflammation (29).

An important link has been found between muscle contraction activity and immune changes, and the notion 
that exercise stimulates the acute increase in cytokines has been demonstrated (16). The IL-6 is produced in the same way in type I and II fibres, while stated that in the 2-hour exercise, IL-6 is produced prominently in type II fibers (30). The combination of muscle fibres is not only one of the main factors in determining the IL-6 response to exercise, but also in determining the amount of mechanical efficiency during fatigue contractions (30). Therefore, one of the reasons for the observed changes in the present study could be the percentage of the use of muscle fibres. Although this point has not been investigated in the present study, the recall of type 2 fibres in resistance training is possible.

IL-6, which plays a metabolic and key role in inflammatory conditions, increases in sports activities more than other cytokines. The metabolic and physiological effects of muscle contraction on cytokine changes are not fully understood, but research has shown that exercise produces IL-6 from a muscle that can have metabolic effects on tissues and other organs (26). Note that the source of their secretion is also very important when measuring inflammatory factors in plasma (31). This means that the substrate involved in the activity can be one of the reasons for the difference in acute inflammatory responses to different types of exercise

Besides, eccentric contractions of resistance training with or without BFR or running on a slope without the use of BFR can play a role in increasing homocysteine and CD54 (ICAM-1) $(3,32,33)$. The ICAM-1 (CD54) has been reported to be affected by physiological stress such as physical activity, and it is associated with the role of sympathetic nerve activity and its effect on immune function and inflammation (34). Therefore, the acute responses of changes in adhesive molecules such as ICAM are likely to be more important than their chronic responses. Because one year of resistance training, despite the improvement in inflammatory levels, did not significantly improve the adhesive molecules response $(1,35)$. Also, even 16 weeks of endurance, resistance, or combined training does not affect resting inflammatory markers but has improved the physiological functions (29).

\subsection{Conclusions}

The significant inflammatory effects of LIRT+BFR have been reported, which has been confirmed by the findings of the present study. However, due to the methodological considerations, it is still very difficult to draw definitive conclusions about the effect of blood flow restriction. Methods of performing BFR training are also very impor- tant, especially in clinical settings. Short-term hypoxia created in BFR training, such as systemic hypoxia, can impair some functions, and this should be controlled for longterm training. Besides, blood pressure monitoring and the extent of damage to peripheral arteries, especially in individuals with risk factors, are important. Based on the principles of adaptability and gradual overload, it is better not to use a sudden increase in intensity. It is necessary to perform special considerations before prescribing BFRtraining. Due to the acute increase in inflammatory responses, appropriate recovery is recommended. Because of the low acute inflammatory response, LIRT+BFR have a similar positive effect as HIRT and can be used in rehabilitation training. Groups interested in resistance training or physiotherapists who use Katsu training in the rehabilitation program should review the results of these studies. Future studies, however, would do well to explore this issue using more comprehensive study groups.

\section{Footnotes}

Authors' Contribution: Mohammad Bani Asadi, Hassan Sharifi: Investigation, methodology, project administration, resources, software. Bahram Abedi, Hoseyn Fatolahi: Investigation, methodology, project administration, resources, software, formal analysis, conceptualization, supervision, data curation, writing - original draft, writing review \& editing.

Conflict of Interests: The authors declare that no conflict of interest exists with this work.

Ethical Approval: The experimental protocol in the present study (based on 2 protocols of the M.S.c thesis) was approved by the ethics committee of Islamic Azad University, Mahallat Branch, Iran (No. 20021404951010 \& 20021444951001). The researchers' Ethics Committee initially approved the experimental procedures and the study protocols, which were fully explained to all the participants.

Funding/Support: There was no funding/support.

Informed Consent: A written consent form was signed after reading and understanding the details of the experiments. The research was also conducted in terms of the principles stated in the Declaration of Helsinki.

\section{References}

1. Olson TP, Dengel DR, Leon AS, Schmitz KH. Changes in inflammatory biomarkers following one-year of moderate resistance training in overweight women. Int J Obes (Lond). 2007;31(6):996-1003. doi: 10.1038/sj.ijo.0803534. [PubMed:17299382]. 
2. Lixandrao ME, Ugrinowitsch C, Berton R, Vechin FC, Conceicao MS, Damas F, et al. Magnitude of muscle strength and mass adaptations between high-load resistance training versus low-load resistance training associated with blood-flow restriction: A systematic review and meta-analysis. Sports Med. 2018;48(2):361-78. doi: 10.1007/s40279017-0795-y. [PubMed: 29043659].

3. Bizheh N, Jaafari M. The effect of a single bout circuit resistance exercise on homocysteine, hs-CRP and fibrinogen in sedentary middle aged men. Iran J Basic Med Sci. 2011;14(6):568-73. [PubMed: 23493183]. [PubMed Central: PMC3586852].

4. Abe T, Kearns CF, Sato Y. Muscle size and strength are increased following walk training with restricted venous blood flow from the leg muscle, Kaatsu-walk training. J Appl Physiol (1985). 2006;100(5):14606. doi: 10.1152/japplphysiol.01267.2005. [PubMed: 16339340].

5. Cook SB, Brown KA, Deruisseau K, Kanaley JA, Ploutz-Snyder LL. Skeletal muscle adaptations following blood flow-restricted training during 30 days of muscular unloading. J Appl Physiol (1985). 2010;109(2):341-9. doi: 10.1152/japplphysiol.01288.2009. [PubMed: 20522734].

6. Cook SB, Scott BR, Hayes KL, Murphy BG. Neuromuscular adaptations to low-load blood flow restricted resistance training. J Sports Sci Med. 2018;17(1):66-73. [PubMed: 29535579]. [PubMed Central: PMC5844210].

7. Christiansen D, Eibye KH, Rasmussen V, Voldbye HM, Thomassen $\mathrm{M}$, Nyberg $\mathrm{M}$, et al. Cycling with blood flow restriction improves performance and muscle $\mathrm{K}(+)$ regulation and alters the effect of anti-oxidant infusion in humans. J Physiol. 2019;597(9):242144. doi: 10.1113/JP277657. [PubMed: 30843602]. [PubMed Central: PMC6487934].

8. Hughes L, Paton B, Rosenblatt B, Gissane C, Patterson SD. Blood flow restriction training in clinical musculoskeletal rehabilitation: a systematic review and meta-analysis. Br J Sports Med. 2017;51(13):1003-11. doi: 10.1136/bjsports-2016-097071. [PubMed: 28259850].

9. Patterson SD, Hughes L, Warmington S, Burr J, Scott BR, Owens J, et al. Blood flow restriction exercise: Considerations of methodology, application, and safety. Front Physiol. 2019;10:533. doi: 10.3389/fphys.2019.00533. [PubMed: 31156448]. [PubMed Central: PMC6530612]

10. Slysz J, Stultz J, Burr JF. The efficacy of blood flow restricted exercise: A systematic review \& meta-analysis. J Sci Med Sport. 2016;19(8):669-75. doi:10.1016/j.jsams.2015.09.005. [PubMed: 26463594].

11. Vincent KR, Vincent HK. Resistance training for individuals with cardiovascular disease. J Cardiopulm Rehabil. 2006;26(4):207-16. quiz 2178. doi:10.1097/00008483-200607000-00002. [PubMed:16926683].

12. Biazon T, Ugrinowitsch C, Soligon SD, Oliveira RM, Bergamasco JG, Borghi-Silva A, et al. The association between muscle deoxygenation and muscle hypertrophy to blood flow restricted training performed at high and low loads. Front Physiol. 2019;10:446. doi: 10.3389/fphys.2019.00446. [PubMed: 31057426]. [PubMed Central: PMC6479177].

13. Mouser JG, Mattocks KT, Buckner SL, Dankel SJ, Jessee MB, Bell ZW, et al. High-pressure blood flow restriction with very low load resistance training results in peripheral vascular adaptations similar to heavy resistance training. Physiol Meas. 2019;40(3):35003. doi: 10.1088/13616579/abod2a. [PubMed: 30840934].

14. Harris NR, Carter PR, Lee S, Watts MN, Zhang S, Grisham MB. Association between blood flow and inflammatory state in a T-cell transfer model of inflammatory bowel disease in mice. Inflamm Bowel Dis. 2010;16(5):776-82. doi: 10.1002/ibd.21126. [PubMed: 19821506]. [PubMed Central: PMC2856723].

15. Harris NR, Carter PR, Yadav AS, Watts MN, Zhang S, Kosloski-Davidson $\mathrm{M}$, et al. Relationship between inflammation and tissue hypoxia in a mouse model of chronic colitis. Inflamm Bowel Dis. 2011;17(3):7426. doi: 10.1002/ibd.21423. [PubMed: 20878754]. [PubMed Central:
PMC3013240].

16. Almada C, Cataldo LR, Smalley SV, Diaz E, Serrano A, Hodgson MI, et al. Plasma levels of interleukin-6 and interleukin-18 after an acute physical exercise: relation with post-exercise energy intake in twins. J Phys iol Biochem. 2013;69(1):85-95. doi: 10.1007/s13105-012-0191-x. [PubMed: 22810957].

17. Andersson J, Jansson JH, Hellsten G, Nilsson TK, Hallmans G, Boman K. Effects of heavy endurance physical exercise on inflammatory markers in non-athletes. Atherosclerosis. 2010;209(2):601-5. doi: 10.1016/j.atherosclerosis.2009.10.025. [PubMed: 19954777].

18. Duzova H, Karakoc Y, Emre MH, Dogan ZY, Kilinc E. Effects of acute moderate and strenuous exercise bouts on IL-17 production and inflammatory response in trained rats. J Sports Sci Med. 2009;8(2):21924. [PubMed: 24149529]. [PubMed Central: PMC3761488].

19. Meyer T, Gabriel HH, Ratz M, Muller HJ, Kindermann W. Anaerobic exercise induces moderate acute phase response. Med Sci Sports Exerc. 2001;33(4):549-55. doi: 10.1097/00005768-200104000-00007. [PubMed: 11283429].

20. Laswati H, Sugiarto D, Poerwandari D, Pangkahila JA, Kimura H Low-intensity exercise with blood flow restriction increases muscle strength without altering hsCRP and fibrinogen levels in healthy subjects. Chin J Physiol. 2018;61(3):188-95. doi: 10.4077/CJP.2018.BAG567. [PubMed: 29962179].

21. Nemet D, Mills PJ, Cooper DM. Effect of intense wrestling exercise on leucocytes and adhesion molecules in adolescent boys Br J Sports Med. 2004;38(2):154-8. doi: 10.1136/bjsm.2002.002576. [PubMed: 15039250]. [PubMed Central: PMC1724796].

22. Freidenreich DJ, Volek JS. Immune responses to resistance exercise. Exerc Immunol Rev. 2012;18:8-41. [PubMed: 22876721].

23. Pober JS, Sessa WC. Inflammation and the blood microvascular system. Cold Spring Harb Perspect Biol. 2014;7(1). a016345. doi: 10.1101/cshperspect.a016345. [PubMed: 25384307]. [PubMed Central: PMC4292166].

24. Ding YH, Young CN, Luan X, Li J, Rafols JA, Clark JC, et al. Exercise preconditioning ameliorates inflammatory injury in ischemic rats during reperfusion. Acta Neuropathol. 2005;109(3):237-46. doi: 10.1007/s00401-004-0943-y. [PubMed: 15616790].

25. Loenneke JP, Wilson GJ, Wilson JM. A mechanistic approach to blood flow occlusion. Int J Sports Med. 2010;31(1):1-4. doi: 10.1055/s-0029 1239499. [PubMed: 19885776].

26. Mendham AE, Donges CE, Liberts EA, Duffield R. Effects of mode and intensity on the acute exercise-induced IL-6 and CRP responses in a sedentary, overweight population. Eur J Appl Physiol. 2011;111(6):103545. doi: 10.1007/s00421-010-1724-z. [PubMed: 21088973].

27. Chadorneshin HT, Golestani A, Jamali F, Shirvan SMM, Sarir H, Eivary SHA. The response of intercellular adhesion molecule- 1 to exhaustive submaximal exercise and its correlation with physiological and anthropometric measures. J Med Life. 2018;11(1):36-41. [PubMed: 29696063]. [PubMed Central: PMC5909944].

28. Esteghamati A, Morteza A, Khalilzadeh O, Anvari M, Noshad S, Zandieh A, et al. Physical inactivity is correlated with levels of quantitative Creactive protein in serum, independent of obesity: results of the national surveillance of risk factors of non-communicable diseases in Iran. J Health Popul Nutr. 2012;30(1):66-72. doi:10.3329/jhpn.v30i1.11278. [PubMed: 22524121]. [PubMed Central: PMC3312361].

29. Libardi CA, De Souza GV, Cavaglieri CR, Madruga VA, Chacon-Mikahil MP. Effect of resistance, endurance, and concurrent training on TNF-alpha, IL-6, and CRP. Med Sci Sports Exerc. 2012;44(1):50-6. doi: 10.1249/MSS.ob013e318229d2e9. [PubMed: 21697747].

30. Banzet S, Koulmann N, Simler N, Birot O, Sanchez H, Chapot R, et al Fibre-type specificity of interleukin-6 gene transcription during muscle contraction in rat: association with calcineurin activity. J Phys- 
iol. 2005;566(Pt3):839-47. doi: 10.1113/jphysiol.2005.089193. [PubMed: 15905211]. [PubMed Central: PMC1464774].

31. Chacon-Salinas R, Serafin-Lopez J, Ramos-Payan R, Mendez-Aragon P, Hernandez-Pando R, Van Soolingen D, et al. Differential pattern of cytokine expression by macrophages infected in vitro with different Mycobacterium tuberculosis genotypes. Clin Exp Immunol. 2005;140(3):443-9. doi: 10.1111/j.1365-2249.2005.02797.x. [PubMed: 15932505]. [PubMed Central: PMC1809389].

32. Simpson RJ, Florida-James GD, Whyte GP, Guy K. The effects of intensive, moderate and downhill treadmill running on human blood lymphocytes expressing the adhesion/activation molecules CD54 (ICAM1), CD18 (beta2 integrin) and CD53. Eur J Appl Physiol. 2006;97(1):109-21. doi:10.1007/s00421-006-0146-4. [PubMed:16506060].

33. Vincent HK, Percival S, Creasy R, Alexis D, Seay AN, Laura Ann Z, et al. Acute effects of enhanced eccentric and concentric resistance exercise on metabolism and inflammation. J Nov Physiother. 2014;4(2). doi: 10.4172/2165-7025.1000200. [PubMed: 26807345]. [PubMed Central: PMC4721556].

34. Goebel MU, Mills PJ. Acute psychological stress and exercise and changes in peripheral leukocyte adhesion molecule expression and density. Psychosom Med. 2000;62(5):664-70. doi: 10.1097/00006842200009000-00010. [PubMed: 11020096].

35. Petridou A, Chatzinikolaou A, Fatouros I, Mastorakos G, Mitrakou $\mathrm{A}$, Chandrinou $\mathrm{H}$, et al. Resistance exercise does not affect the serum concentrations of cell adhesion molecules. Br J Sports Med. 2007;41(2):76-9. discussion 79. doi: 10.1136/bjsm.2006.031047. [PubMed: 17127720]. [PubMed Central: PMC2658932]. 